\title{
200 \\ Solution of Non-linear Equations using Bisection Method by New Technical Method
}

\author{
By \\ Subhi Abdalazim Aljily Osman \\ Department of Mathematics , Faculty of Computer Science and \\ Information Technology, University of ALBUTANA, Rufaa Sudan \\ Abdel Radi Abdel Rahman Abdel Gadir Abdel Rahman \\ Department of Mathematics, Faculty of Education, Omdurman \\ Islamic University, Omdurman, Sudan \\ Adam Osman Ali Mohammed \\ Department of Mathematics, Faculty of Engineering, Sennar \\ University, Sennar, Sudan \\ Abualez Alamin Ahmed Ali
}

Department of Mathematics, Faculty of Education, University of Holly Quran and Tassel of Science, Rufaa, Sudan

Doi: 10.21608/jasep.2022.216291

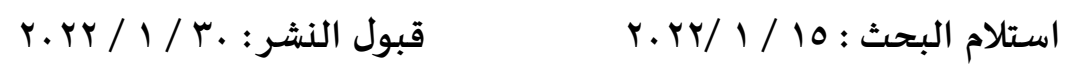

Osman ,Subhi Abdalazim Aljily \& Abdel Rahman, Abdel Radi Abdel Rahman Abdel Gadir \& Mohammed, Adam Osman Ali \& Ali, Abualez Alamin Ahmed (2022). Solution of Non-linear Equations using Bisection Method by New Technical Method, 6 (26), The Arab Journal of Educational and Psychological Sciences, Arab Institute for Education, Science and Arts, Egypy, 261-274.

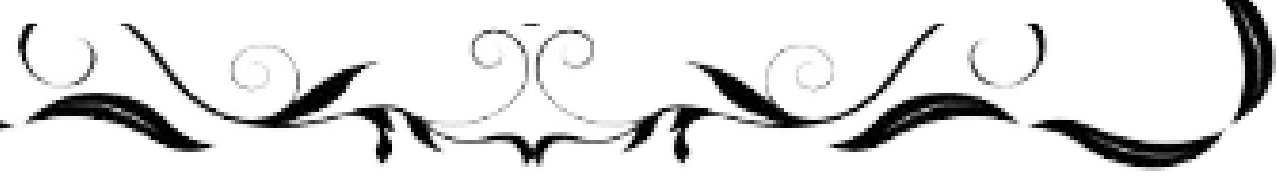




\section{Solution of Non-linear Equations using. Subhi Abdalazim et al.}

\section{Solution of Non-linear Equations using Bisection Method by New Technical Method}

\section{Abstract:}

Numerical approximation of the root-finding problem its important tool for process involves finding a root, or solution of nonlinear equation of the form $f(x)=0$, for a given function $\mathrm{f}$. A root of this equation is also called a zero of the function $f$.When we implementing the method on a computer we need to consider the effects of round-off error. For example the computation of the midpoint of the interval $\left[a_{n}, b_{n}\right]$ should be found from the equation. The Bisection method is used to determine to any specified accuracy that your computer will permit a solution to $f(x)=0$ on an interval $[a, b]$, provided that $f$ is continuous on the interval and that $f(a)$ and $f(b)$ are of opposite sign. Although the method will work for the case when more than one root is contained in the interval $[a, b]$, we assume for simplicity of our discussion that the root in this interval is unique. the method stops if one of the midpoints happens to coincide with the root. It also stops when the length of the search interval is less than some prescribed tolerance. The having method is characterized by the fact that it always includes convergence of the individual islands. It is also characterized by the case of calculating errors, but one of its disadvantages is that it is slow to converge to reach the solution. To compare with the a new technical method of the solution. We followed applied numerical method using a new technical method in computer and we found that the new technical method of solution is much faster and more accurate.

Keywords : Solution, Non-linear Equations , Bisection Method, New Technical Method.

\section{Introduction:}

Non-linear equations arise in most disciplines including computer science, natural sciences (civil engineering, electrical engineering, mechanical engineering), biological engineering and social sciences 


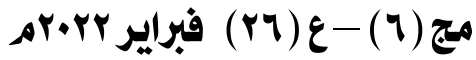

\section{المجلة العربية للعلور التربوية والنفسية}

(psychology, economics), etc. Problems such as minimization, target shooting, orbital motion, plenary motion, etc., often lead to finding roots of non-linear functional equations. There are classical rootfinding algorithms: bisection, false position, Newton-Raphson, modified Newton-Raphson, secant and modified secant method, for finding roots of a non-linear equation $f(x)=0[1]$. Many bracketing algorithms have been described in literature for finding a single root of a nonlinear equation $f(x)=0$. The most basic bracketing method is a dichotomy method also known as a bisection method with a rather slow convergence[2]. Bisection method and main results The goal of the bisection method which is studied in this paper is to locate and to approximate the zeros of an analytic function $f$ in a specified bounded domain [3].

\section{Solving Nonlinear Equations:}

Given a function $f: R \rightarrow R$, we seek a point $x_{m} \in R$ such that $f\left(x_{m}\right)=0$. This $x_{m}$ is called a root of the equation $f(x)=0$, or simply a zero of $f$. At first, we only require that $\mathrm{f}$ be continuous an interval $[a, b]$ of the real line, $f \in C[a, b]$, and that this interval contains the root of interest. The function $f$ could have many different roots; we only look for one. In practice, $f$ could be quite complicated (e.g., evaluation of a parameter dependent integral or differential equation) that is expensive to evaluate (e.g., requiring minutes, hours, ... ), so we seek algorithms that produce a solution that is accurate to high precision while keeping evaluations of $f$ to a minimum [4].

\section{Definitions and Sources of Error:}

We now give a rough classification of the major ways in which error is introduced into the solution of a problem, including some that fall outside the usual scope of mathematics. We begin with a few simple definitions about error.In solving a problem, we seek an exact or true solution, which we denote by

$X_{T}$-Approximations are usually made in solving the problem, resulting in an approximate solution $X_{A}$. We define the error in $X_{A}$ by 


\section{Solution of Non-linear Equations using. Subhi Abdalazim et al.}

$$
\operatorname{Error}\left(X_{A}\right)=\operatorname{Error} X_{A}=X_{T}-X_{A}
$$

For many purposes, we prefer to study the percentage or relative error in $X_{A}$.

$$
\operatorname{Rel}\left(X_{A}\right) \equiv \text { relative error in } X_{A}=\frac{X_{T}-X_{A}}{X_{T}}
$$

Provided $X_{T} \neq 0$. This has already been referred to in $X_{A} \frac{x-f(x)}{x} \equiv \varepsilon$ in measuring the error in $f(x)$ [5].

\section{Bracketing Methods:}

In the rest of the text, let $\mathrm{f}$ be a real and continuous function on an interval $[a, b], f(a)$ and $f(b)$ have different parity i.e. $f(a) f(b)<$ 0 . Therefore, there is at least one real root $r$ in the interval $[a, b]$ of the equation $f(x)=0[6]$.

\section{Bisection Method:}

This method is based if a function $f(x)$ is continuous between $a$ and $b$ and $f(a)$ and $f(b)$ are of opposite signs then there exists at least one root between $a$ and $b$. For definition let $f(a)$ be negative and $f(b)$ be positive [7 ].

We first consider the Bisection (Binary search) Method which is based on the Intermediate Value Theorem (IVT). IVT Illustration Suppose a continuous function, defined on $[a, b]$ is given with $f(a)$ and $f(b)$ of opposite sign. By the IVT, there exists a point $p \in$ $(a, b)$ for which $f(p)=0$. In what follows, it will be assumed that the root in this interval is unique Suppose $f$ is a continuous function defined on the interval $[a, b]$, with $f(a)$ and $f(b)$ of opposite sign. The Intermediate Value Theorem implies that a number $\mathrm{p}$ exists in $(a, b)$ with $f(p)=0$. Although the procedure will work when there is more than one root in the interval $(a, b)$, we assume for simplicity that the root in this interval is unique. The method calls for a repeated halving (or bisecting) of subintervals of $[a, b]$ and, at each step, locating the half containing $p[8]$.

Example (5.1): Find the toot of the equation $x^{3}-2 x-5=0$ using Bisection method hence the root lies between 2 and 3 


\section{مج (Y) - (Y (Y ) فبراير r.r.}

\section{المجلة العربية للعلوم التربوية والنفسية}

\section{Solution:}

hence the root lies between 2 and 3

$$
f(2)=-1 \text { and } f(3)=16
$$

$$
x_{1}=\frac{2+3}{2}=2.5
$$

The root lies between 2 and 2.5

Since $f\left(x_{1}\right)=f(2.5)=5.6250$,

The root lies between 2 and 2.25

$$
x_{2}=\frac{2+2.5}{2}=2.25
$$

Now, $f\left(x_{2}\right)=f(2.25)=1.890625$,

$$
x_{3}=\frac{2+2.25}{2}=2.125
$$

The root lies between 2 and 2.125

Since $f\left(x_{3}\right)=f(2.125)=0.3457$,

$$
x_{4}=\frac{2+2.125}{2}=2.0625
$$

Proceeding in this way, we obtain the successive approximations:

We find

$$
\begin{array}{cc}
x_{5}=2.09375, & x_{6}=2.10938 \\
x_{7}=2.10156 & x_{8}=2.09766 \\
x_{9}=2.09570 & x_{10}=2.09473 \\
x_{11}=2.09424 & \ldots \ldots \ldots \ldots \ldots
\end{array}
$$

and

$$
x_{11}-x_{10}=-0.0005
$$

$$
\left|\frac{x_{11}-x_{10}}{x_{11}}\right| \times 100=\frac{0.0005}{2.09424} \times 100=0.02 \%
$$

Hence a root, correct to three decimal places is 2.094[7].

Example (5.2) Show that $f(x)=x^{3}+4 x^{2}-10=0$ has a root in $[1,2]$, and use the Bisection method to determine an approximation to the root that is accurate to at least within $10^{-4}$. 


\section{Solution of Non-linear Equations using. Subhi Abdalazim et al.}

\section{Solution:}

Because $f(1)=-5$ and $f(2)=14$ ensures that this continuous function has a root in $[1,2]$.

For the first iteration of the Bisection method we use the fact that at the midpoint of $[1,2]$ we have $f(1.5)=2.375>0$. This indicates that we should select the interval $[1,1.5]$ for our second iteration. Then we find that $f(1.25)=-1.796875$ so our new interval becomes[1.25,1.5], whose midpoint is 1.375 . Continuing in this manner gives the values in Table (5.1). After 13 iterations, $p_{13}=$ 1.365112305 approximates the rootpwith an error

$$
\begin{gathered}
\left|p-p_{13}\right|<\left|b_{14}-a_{14}\right|=|1.365234375-1.365112305| \\
=0.000122070 .
\end{gathered}
$$

Since $\left|a_{14}\right|<|p|$,wehave

Table N0. (5.1):

$$
\frac{\left|p-p_{13}\right|}{|p|}<\frac{\left|b_{14}-a_{14}\right|}{\left|a_{14}\right|} \leq 9.0 \times 10^{-5}
$$

\begin{tabular}{|c|c|c|c|c|}
\hline $\mathrm{n}$ & $a_{n}$ & $b_{n}$ & $p_{n}$ & $f\left(p_{n}\right)$ \\
\hline 1 & 1.0 & 2.0 & 1.5 & 2.375 \\
\hline 2 & 1.0 & 1.5 & 1.25 & -1.79687 \\
\hline 3 & 1.25 & 1.5 & 1.375 & 0.16211 \\
\hline 4 & 1.25 & 1.375 & 1.3125 & -0.84839 \\
\hline 5 & 1.3125 & 1.375 & 1.34375 & -0.35098 \\
\hline 6 & 1.34375 & 1.375 & 1.359375 & -0.09641 \\
\hline 7 & 1.359375 & 1.375 & 1.3671875 & 0.03236 \\
\hline 8 & 1.359375 & 1.3671875 & 1.36328125 & -0.03215 \\
\hline 9 & 1.36328125 & 1.3671875 & 1.365234375 & 0.000072 \\
\hline 10 & 1.36328125 & 1.365234375 & 1.364257813 & -0.01605 \\
\hline 11 & 1.364257813 & 1.365234375 & 1.364746094 & -0.00799 \\
\hline 12 & 1.364746094 & 1.365234375 & 1.364990235 & -0.00396 \\
\hline 13 & 1.364990235 & 1.365234375 & 1.365112305 & -0.00194 \\
\hline
\end{tabular}

So the approximation is correct to at least within $10^{-4}$. The correct value of $p$ to nine decimal places is $p=1.365230013$. Note that $p_{9}$ is closer to $p$ than is the final approximation $p_{13}$. You might suspect 
this is true because $\left|f\left(p_{9}\right)\right|<\left|f\left(p_{13}\right)\right|$, but we cannot be sure of this unless the true answer is known.

The Bisection method, though conceptually clear, has significant drawbacks. It is relatively slow to converge (that is, $N$ may become quite large before $\left|p-p_{N}\right|$ is sufficiently small), and a good intermediate approximation might be inadvertently discarded. However, the method has the important property that it always converges to a solution, and for that reason it is often used as a starter for the more efficient methods .[8].

Example(5.3): Use the bisection method to approximate the value of $\sqrt{5}$ within 0.00001 accuracy.

\section{Solution:}

Finding the square root of 5 is equivalent to finding the roots of $x^{2}-5=0$ We expect the positive root to be in the $2<x<3$ interval so we assign $x_{1}=2 \quad x_{2}=3$. The average of these values is $x_{m}=2.5$ we can use it with any polynomial equation. [9]

Table No. (5.2):

\begin{tabular}{|c|c|c|c|c|c|c|}
\hline $\mathrm{n}$ & $x_{1}$ & $x_{2}$ & $x_{m}$ & $f\left(x_{1}\right)$ & $f\left(x_{21}\right)$ & $f\left(x_{m}\right)$ \\
\hline 1 & 2.00000 & 3.00000 & 2.50000 & -1.00000 & 1.25000 & -1.25000 \\
\hline 2 & 2.00000 & 2.50000 & 1.25000 & -1.00000 & 0.06250 & -0.06250 \\
\hline 3 & 2.00000 & 2.25000 & 2.12500 & -1.00000 & -0.48438 & 0.48438 \\
\hline 4 & 2.12500 & 2.25000 & 2.18750 & -0.48438 & -0.21484 & 0.10406 \\
\hline 5 & 2.18750 & 2.25000 & 2.21875 & -0.21484 & -0.07715 & 0.01657 \\
\hline 6 & 2.21875 & 2.25000 & 2.23438 & -0.07715 & -0.00757 & 0.00058 \\
\hline 7 & 2.23438 & 2.25000 & 2.24219 & -0.00757 & 0.02740 & -0.00021 \\
\hline 8 & 2.23438 & 2.24219 & 2.23828 & -0.00757 & 0.00990 & -0.00007 \\
\hline 9 & 2.23438 & 2.23828 & 2.23633 & -0.00757 & 0.00116 & -0.00001 \\
\hline 10 & 2.23438 & 2.23633 & 2.23535 & -0.00757 & -0.00320 & 0.00002 \\
\hline 11 & 2.23535 & 2.23633 & 2.23584 & -0.00320 & -0.00102 & 0.00000 \\
\hline 12 & 2.23584 & 2.23633 & 2.23608 & -0.00102 & 0.00007 & 0.00000 \\
\hline 13 & 2.23584 & 2.23608 & 2.23596 & -0.00102 & -0.00047 & 0.00000 \\
\hline 14 & 2.23596 & 2.23608 & 2.23602 & -0.00047 & -0.00020 & 0.00000 \\
\hline 15 & 2.23602 & 2.23608 & 2.23605 & -0.00020 & -0.00006 & 0.00000 \\
\hline
\end{tabular}


Solution of Non-linear Equations using. Subhi Abdalazim et al.

\begin{tabular}{|l|l|l|l|l|l|l|}
\hline 16 & 2.23605 & 2.23608 & 2.23607 & -0.00006 & 0.00000 & 0.00000 \\
\hline 17 & 2.23605 & 2.23607 & 2.23606 & -0.00006 & -0.00003 & 0.00000 \\
\hline 18 & 2.23606 & 2.23607 & 2.23606 & -0.00003 & -0.00001 & 0.00000 \\
\hline
\end{tabular}

\section{Computational Steps:}

To begin, set $\mathrm{a}_{1}=a$ and $\mathrm{b}_{1}=b$ and let $\mathrm{p}_{1}$ be the midpoint of [a, b]; that is,

$$
\mathrm{p}_{1}=\frac{\mathrm{a}_{1}+\mathrm{b}_{1}}{2}
$$

If $f\left(\mathrm{p}_{1}\right)=0$, then $\mathrm{p}=\mathrm{p}_{1}$, and we are done.

If $f\left(\mathrm{p}_{1}\right) \neq 0$, then $f\left(\mathrm{p}_{1}\right)$ has the same sign as either $f(a)$ or $f(b)$. If $f\left(\mathrm{p}_{1}\right)$ and $f\left(\mathrm{a}_{1}\right)$ have the same sign $P\left(P, \mathrm{~b}_{1}\right)$ set $\mathrm{a}_{2}=\mathrm{p}_{1}$ and $\mathrm{b}_{2}=\mathrm{p}_{1}$ Then re-apply the process to the interval $\left[\mathrm{a}_{2} \mathrm{~b}_{2}\right]$, ect

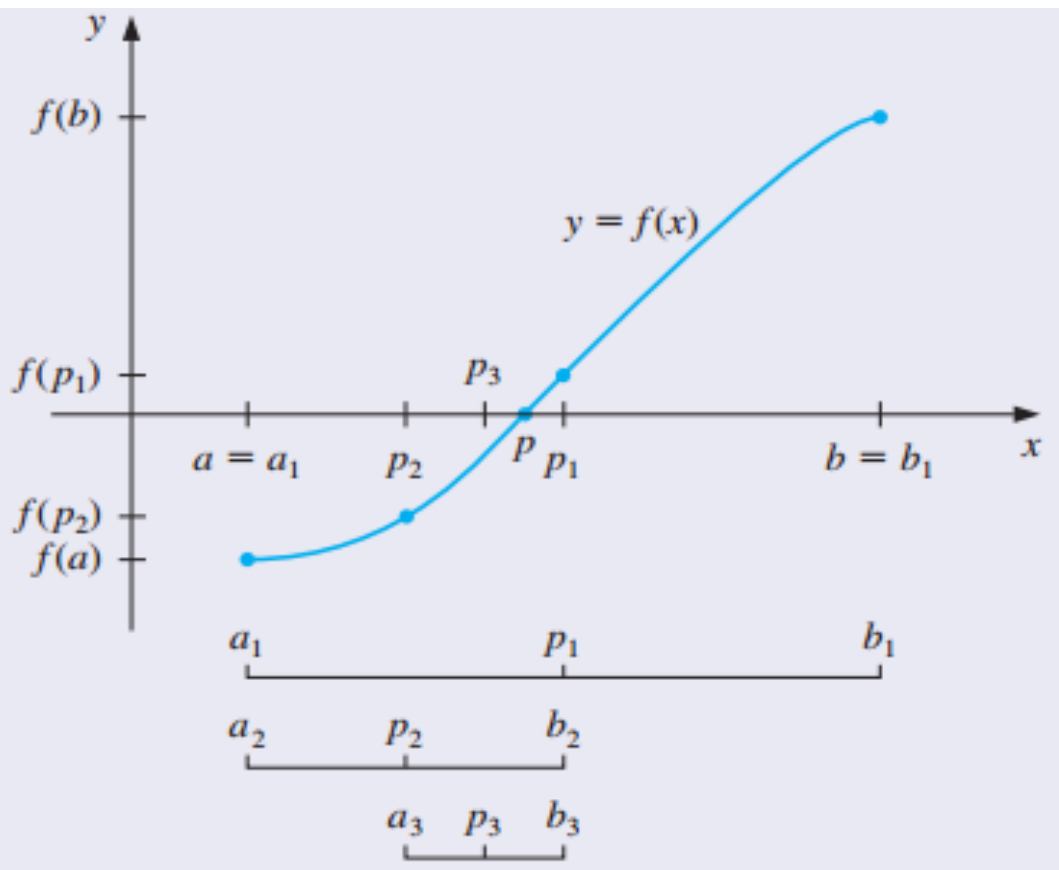

Fig No (1) Interval Halving to Bracket the Root

To find a solution to $f(x)=0$ given the continuous function $f$ on the interval $[a, b]$, where $f(a)$ and $f(b)$ have opposite signs: 


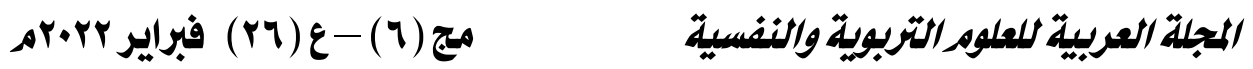

INPUT endpoints $a, b$; to lerance TOL; maximum number of iterations $N_{0}$

OUTPUT approximate solution $\mathrm{p}$ or message of failure.

Step 1 Set $i=1$;

$F A=f(a)$.

Step 2 While $\leq N_{0}$ do Steps 3-6.

Step 3 set $\mathrm{p}=a+(b-a) / 2$; (Compute pi.)

$F P=f(\mathrm{p})$.

Step 4 if $F P=0$ or $(b-a) / 2<T O L$ then

OUTPUT (p); (Procedure completed successfully.)

STOP.

Step 5 Set $i=i+1$.

Step 6 if FA.FP $>0$ then set $a=b$; (Compute $a, b)$

$F A=F P$

Else setb=P.(FA is unchanged)

Step 7 OUTPUT (Method failed after $N_{0}={ }^{\prime} N_{0}$ );

(the procedure was unsuccessful.) STOP.

Other stopping procedures can be applied in Step 4. For example, we can select a tolerance $\varepsilon>0$ and generate $\mathrm{p}_{1}, \ldots, \mathrm{p}_{N}$ until one of the following conditions is met:

$\left|\mathrm{f}\left(\mathrm{p}_{N}\right)\right|<\varepsilon[8]$

$$
\begin{gathered}
\left|\mathrm{p}_{N}-\mathrm{p}_{N-1}\right|<\varepsilon \\
\frac{\left|\mathrm{p}_{N}-\mathrm{p}_{N-1}\right|}{\left|\mathrm{p}_{N}\right|}<\varepsilon \quad \mathrm{p}_{N} \neq 0 \quad \text { or }
\end{gathered}
$$

Example (6.1) Show that $f(x)=x^{3}+4 x^{2}-10=0$ has a root in $[1,2]$, and use the Bisection method to determine an approximation to the root that is accurate to at least within $10^{-4}$ using Matlab.[8]

\section{Solution:}

You must first know the function in $m$.file 


\section{Solution of Non-linear Equations using. Subhi Abdalazim et al.}

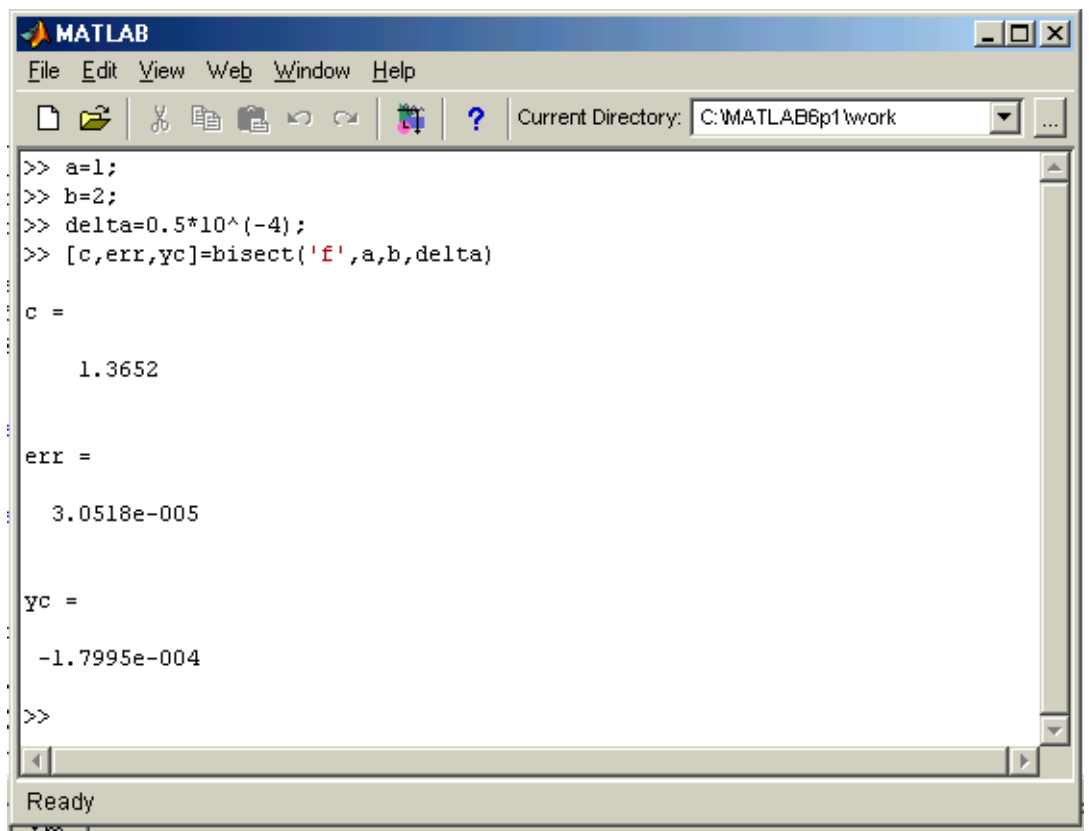

Second: In the Command Window we write inputs

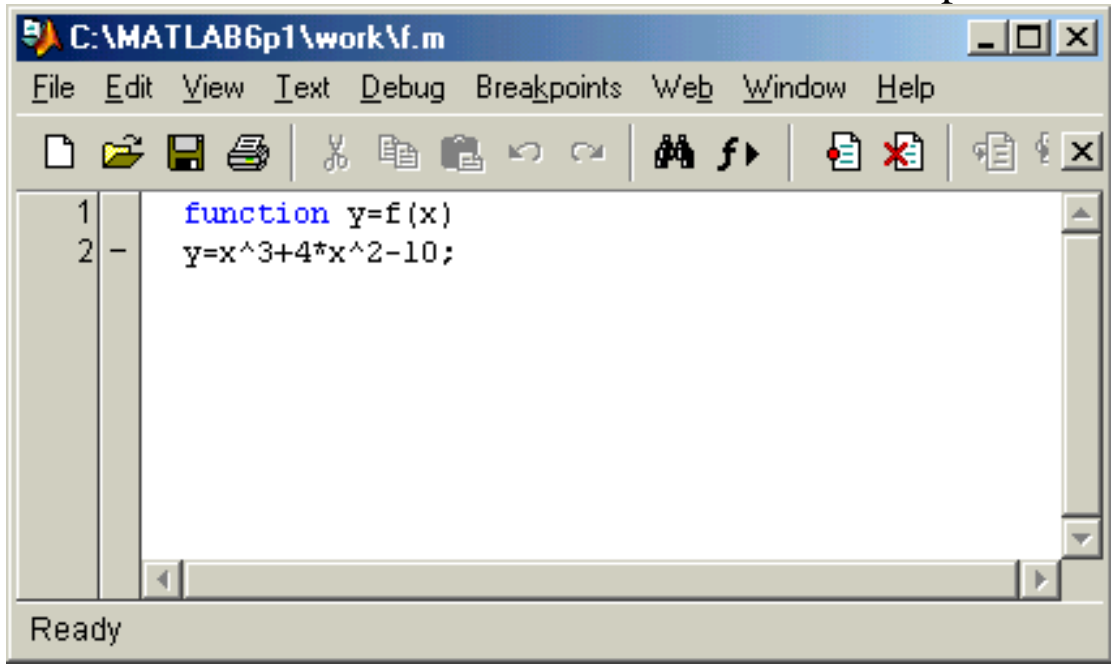


Example (5.2): Use the bisection method with an Excel spreadsheet to approximate the value of $\sqrt{5}$ within 0.00001 accuracy using Matlab.[9]

\section{Solution:}

Finding the square root of 5 is equivalent to finding the roots of $x^{2}-5=0$ We expect the positive root to be in the $2<x<3$ interval so we assign $x_{1}=2 \quad x_{2}=3$. The average of these values is $x_{m}=2.5$ we can use it with any polynomial equation.

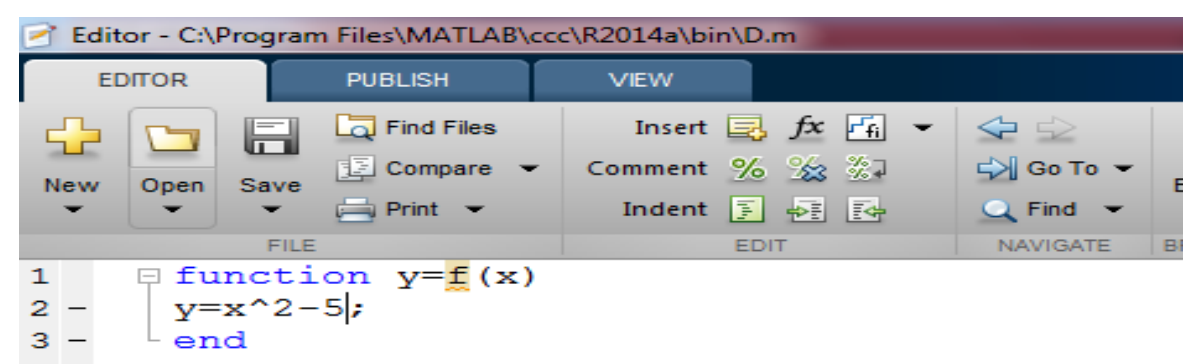




\section{Solution of Non-linear Equations using. Subhi Abdalazim et al.}

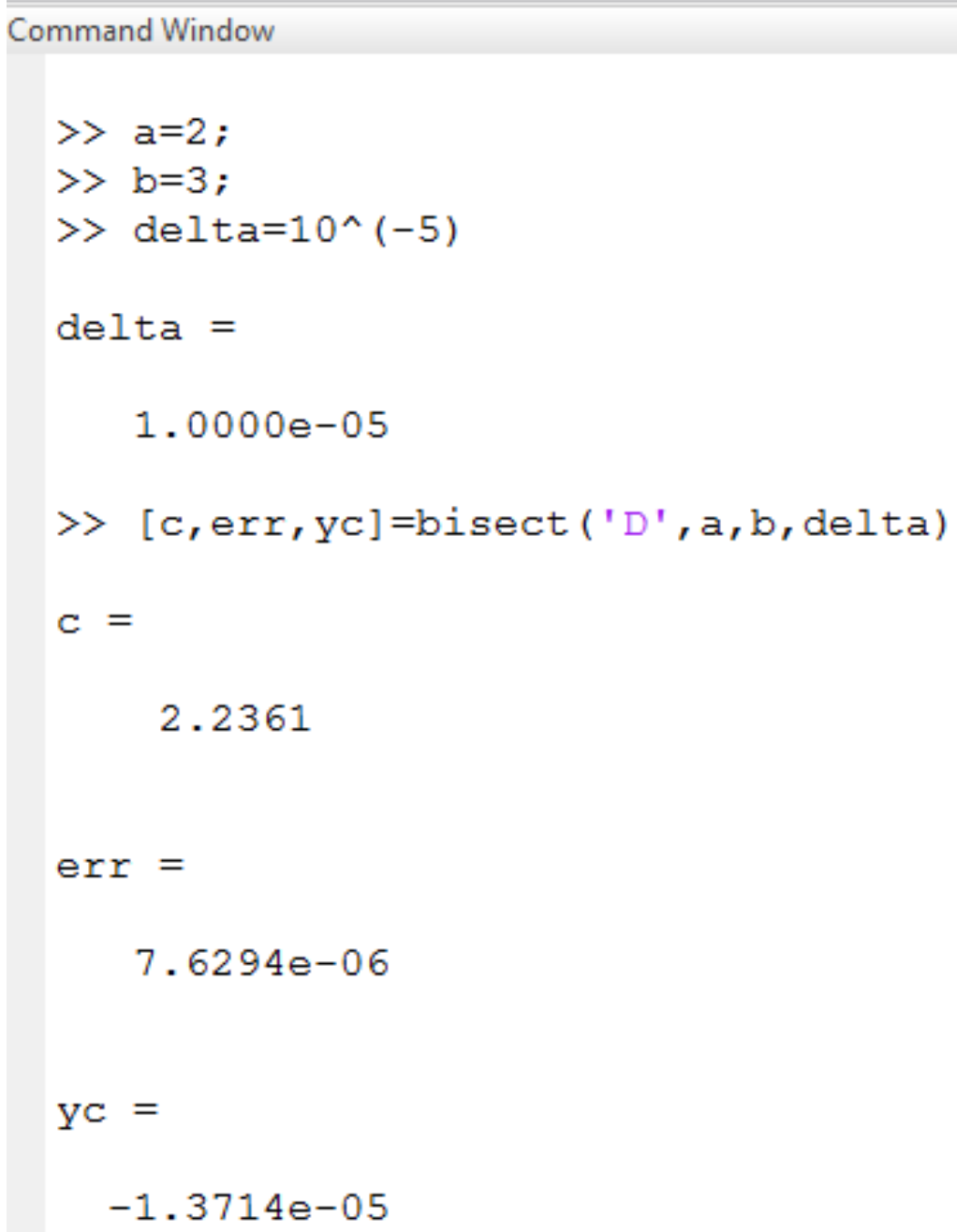

\section{Matlab Code :}

Function [c,err,yc] $=$ bisect(f,a,b,delta)

\%Input - $\mathrm{f}$ is the function input as a string ' $\mathrm{f}$ '

$\% \quad-\mathrm{a}$ and $\mathrm{b}$ are the left and right end points

$\% \quad$-delta is the tolerance

$\%$ Output $-\mathrm{c}$ is the zero

$\% \quad-\mathrm{yc}=\mathrm{f}(\mathrm{c}($ 
$\% \quad$-err is the error estimate for $\mathrm{c}$

$\mathrm{ya}=\mathrm{feval}(\mathrm{f}, \mathrm{a})$;

$\mathrm{yb}=\mathrm{feval}(\mathrm{f}, \mathrm{b})$;

ifya* $\mathrm{yb}>0$

end

$\max 1=1+$ round $((\log (\mathrm{b}-\mathrm{a})-\log ($ delta $)) / \log (2))$;

for $\mathrm{k}=1: \max 1$

$\mathrm{c}=(\mathrm{a}+\mathrm{b}) / 2$;

$\mathrm{yc}=\mathrm{feval}(\mathrm{f}, \mathrm{c})$;

ifyc $==0$

$\mathrm{a}=\mathrm{c}$;

$\mathrm{b}=\mathrm{c}$;

elseifyb*yc $>0$

$\mathrm{b}=\mathrm{c}$;

$\mathrm{yb}=\mathrm{yc}$;

else

$\mathrm{a}=\mathrm{c}$;

$\mathrm{ya}=\mathrm{yc}$;

end

if $\mathrm{b}$-a $<$ delta,break,end

end

$\mathrm{c}=(\mathrm{a}+\mathrm{b}) / 2$;

err=abs(b-a);

$\mathrm{yc}=\mathrm{feval}(\mathrm{f}, \mathrm{c})$;

\section{Conclusion:}

The goal of the bisection method which is studied in this paper is to locate the method stops if one of the midpoints happens to coincide with the root 


\section{Solution of Non-linear Equations using. Subhi Abdalazim et al.}

\section{Reference :}

[1] ChamanLalSabharwal, Blended Root Finding Algorithm Outperforms Bisection and RegulaFalsi Algorithms, Published: 16 November 2019

[2] AlojzSuhadolnik, Combined bracketing methods for solving nonlinear equations, Volume 25, Issue 11, November 2012

[3] J.-C. Yakoubsohn, Numerical analysis of a bisection-exclusion method to find zeros of univariate analytic functions, , 21 June 2005

[4] M. Embree, Rice University, NUMERICAL ANALYSIS , 29 November 2009.

[5] Kendall E. Atkinson, John Wiley \& Sons, AN INTRODUCTION TO NUMERICAL ANALYSIS , Second Edition, 1978

[6] SomkidIntep, A Review of Bracketing Methods for Finding Zeros of Nonlinear Functions, Applied Mathematical Sciences, Vol. 12, 2018 [7] S.S .SASTRY. Introductory methods of numerical analysis PHI Learning private limited New Delhi-110001, fifth Edition 2012.

[8] Richard L. Burden, J. Douglas Faires, Numerical Analysis, Ninth Edition, 2011

[9] Steven T. Karris, Numerical Analysis, Second Edition, 2004 